\title{
Synchronous multi-scale observations on rock damage and rupture
}

\author{
X.H. Xu ${ }^{\text {a }}$, S.P. Ma ${ }^{\text {b }}$, M.F. Xia ${ }^{\text {a,c }}$, F.J. Ke ${ }^{\text {d,a }}$, Y.L. Bai ${ }^{\text {a,* }}$ \\ a State Key Laboratory of Nonlinear Mechanics (LNM), Institute of Mechanics, \\ Chinese Academy of Sciences, Beijing 100080, PR China \\ b Department of Geophysics, Peking University, Beijing 100871, PR China \\ ${ }^{\mathrm{c}}$ Department of Physics, Peking University, Beijing 100871, PR China \\ d Department of Applied Physics, BeiHang University, Beijing 100088, PR China
}

Available online 2 September 2005

\begin{abstract}
This paper reports a multi-scale study on damage evolution process and rupture of gabbro under uniaxial compression with several experimental techniques, including MTS810 testing machine, white digital speckle correlation method, and acoustic emission technique. In particular, the synchronization of the three experimental systems is realized for the study of relationship of deformation and damage at multiple scales. It is found that there are significant correlation between damage evolution at small and large length scales, and rupture at sample scale, especially it displays critical sensitivity at multiple scales and trans-scale fluctuations.
\end{abstract}

(C) 2005 Elsevier Ltd. All rights reserved.

\section{Introduction}

Rock is a multi-scale heterogeneous brittle material. It is well established that the rupture of heterogeneous brittle media in compression is due to generation, growth and coalescence of microcracks [1-4], which will induce damage localization at locally macroscopic scale and catastrophic rupture at macroscopic scale. That is to say, the failure

\footnotetext{
* Corresponding author.

E-mail address: baiyl@Inm.imech.ac.cn (Y.L. Bai).
}

process usually takes place through a sequence from small scale to large scale and eventually to the whole sample scale [5]. During such a process, the effects of some disordered structures at mesoscopic scales can be amplified significantly due to dynamical non-linearity, and become crucial for eventual rupture. Therefore, to better understand damage and rupture behavior of rock, it is necessary to observe the process at different scales synchronously.

The damage evolution process and rupture of gabbro sample under uniaxial compression is studied. Several experimental techniques are combined 
to synchronously observe the process of rock damage and rupture at different scales.

(1) The gabbro sample is loaded under uniaxial compression with a MTS810 testing machine. The experimental nominal stressstrain curve provides a description of global behavior at sample scale.

(2) The surface image series is captured by a CCD camera during the loading process. A speed-shift image capture method according to loading status of the sample is provided for effective use of resource.

After experiment, the speckle images are analyzed with digital speckle correlation method (DSCM) [6,7] and the deformation field series during the loading procedure is obtained. Based on coarse-grained analysis, the responses at length scales ranging from about $10^{-5}$ sample scale to sample scale are obtained.

(3) Acoustic emission (AE) [8,9] signals are elastic waves resulting from crack initiation and propagation in material. Therefore, by making use of AE monitoring system, acoustic emission associates with microcracks were recorded. The detection of $\mathrm{AE}$ signals enables us to characterize micro-damage on mesoscopic scale statistically.

Most importantly, in order to compare the responses at different scales, the synchronization of the recordings made in the three systems should be realized in an experiment. Then, a synchronous multi-scale analysis on damage and rupture of rock can be performed to provide a complete and clear insight of the process.

\section{Experimental details}

\subsection{Measurement of nominal stress-strain curve}

The experimental system is illustrated in Figs. 1 and 2 . In our tests, rectangular gabbro samples, $5 \times 5 \times 13 \mathrm{~mm}^{3}$, were compressed uniaxially with a servo-hydraulic testing machine (MTS810). The resolution of the load sensor is $10 \mathrm{~N}$ in the range

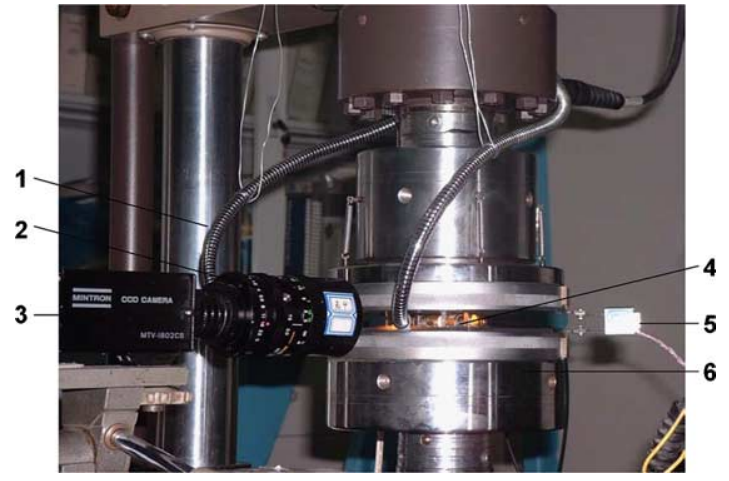

Fig. 1. The experimental set-up for observing rock damage and rupture, 1-lumination, 2-camera, 3- CCD , 4-AE sensors and rock sample, 5-extensometer, 6-platen of MTS810.

of $20 \mathrm{kN}$, and the resolution of displacement sensor is $20 \mu \mathrm{m}$ in the range of $\pm 10 \mathrm{~mm}$. Due to the rigidity of the testing machine, the displacement recorded by the MTS810 displacement sensor, which is called boundary displacement later, is not the real displacement of the rock sample. Thus, the displacement of the sample was measured by an extensometer with resolution of $3 \mu \mathrm{m}$ and an offset of load $1 \mathrm{kN}$. The loading mode was load control till $0.8 \mathrm{kN}$ and then held $1 \mathrm{~min}$, afterwards the mode changed to boundary displacement control with velocity of $0.02 \mathrm{~mm} / \mathrm{min}$. Fig. 3 shows the curves of load versus displacement, respectively obtained by extensometer (solid line) and MTS810 displacement sensor (dashed line), of a gabbro sample under uniaxial loading. In such a loading system, the boundary displacement is the external controlling variable, while the nominal stress and nominal strain are the responses of the rock sample at sample scale.

\subsection{Surface image capture and strain field calculation}

For speckle correlation analysis, one surface of the rock sample was first spray-painted to make artificial speckle (see Fig. 4). During loading, the surface of the sample was illuminated by two fiber optic white light sources, and consecutive surface images were captured and transferred to computer 


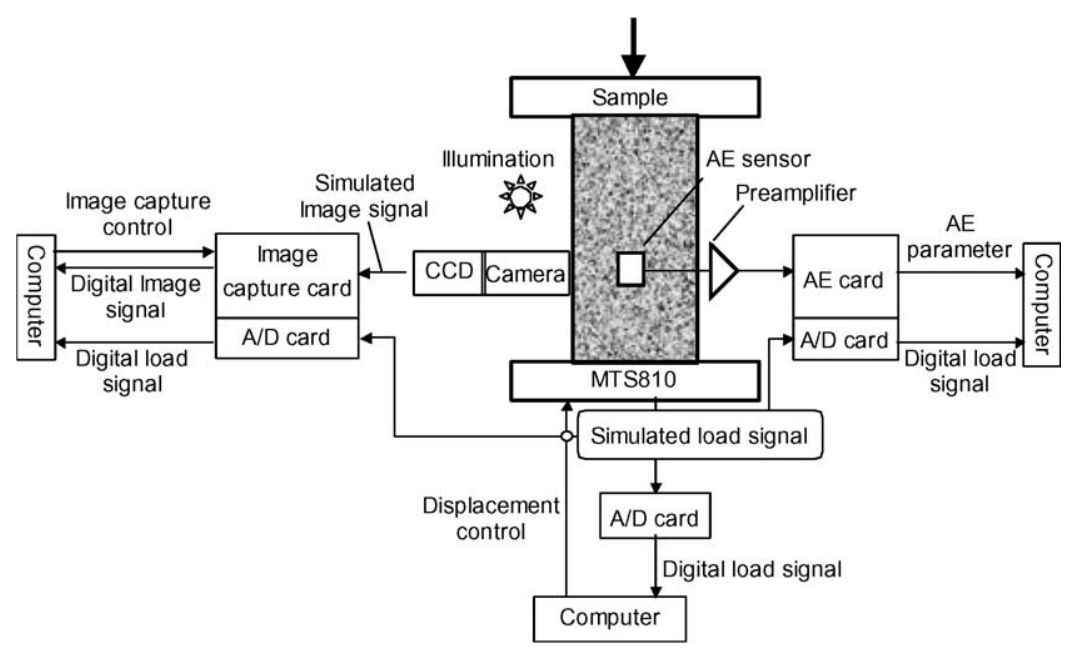

Fig. 2. Schematic diagram of experimental set-up.

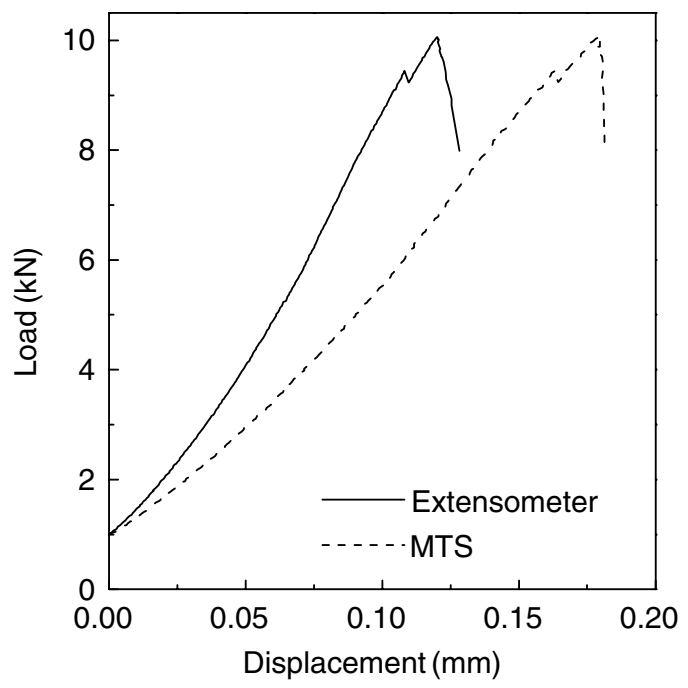

Fig. 3. Curves of load versus displacement obtained by extensometer (solid line) and MTS810 displacement sensor (dashed line), respectively, for a gabbro sample under uniaxial loading.

by a video camera with a CCD array of $768 \times$ 576 pixels. The whole sample surface was mapped to approximately 480 pixels in loading direction (axis 2 in Fig. 4) and 180 pixels in the direction (axis 1 in Fig. 4) vertical to loading. Thus, the length-pixel ratio of the imaging system is about $28 \mu \mathrm{m} /$ pixel.
Later, we select a calculating domain with 401 pixels in axis 2 and 131 pixels in axis 1, i.e. totally 52531 calculating points. Then, the digital speckle correlation method was carried out, and both displacement and strain fields of the sample surface during the loading process were obtained. The contour map of strain field $\varepsilon_{11}^{*}$ is shown in Fig. 5. The obtained displacement and strain of a point can be understood as an average over area of $28 \times 28 \mu \mathrm{m}^{2}$, i.e. approximately $10^{-5}$ sample scale, which is assumed to be locally macroscopic scale in this paper. In addition, the accuracy for displacement measurement is 0.1 pixels in the experiment based on our calibration.

\subsection{Acoustic emission detection}

Moreover, two AE sensors (10 $\mathrm{mm}$ in diameter) were fixed on two sides of a sample with a specially designed clamp. The resonant frequencies of the sensors are $140 \mathrm{kHz}$ and $250 \mathrm{kHz}$ respectively. The sensor output was amplified by $40 \mathrm{~dB}$ at preamplifier and $10 \mathrm{~dB}$ at main amplifier. Then, the AE signals associating with micro-cracks on mesoscopic scale were analyzed on the AE main board produced by the Institute of Computer Technology of Shenyang, and then the AE parameters, such as AE counts, energy, etc., can be obtained. The AE counts and energy series during loading are shown in Fig. 6. 


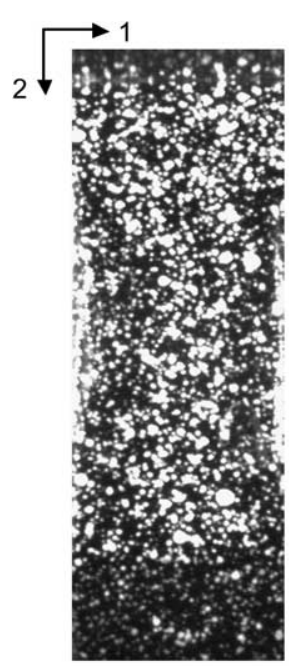

A1

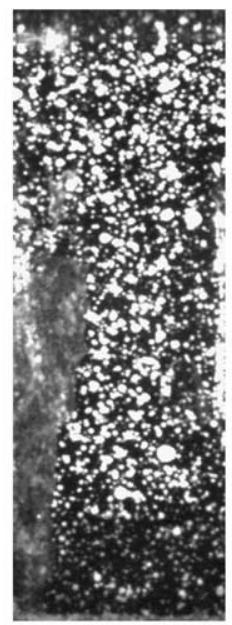

A5

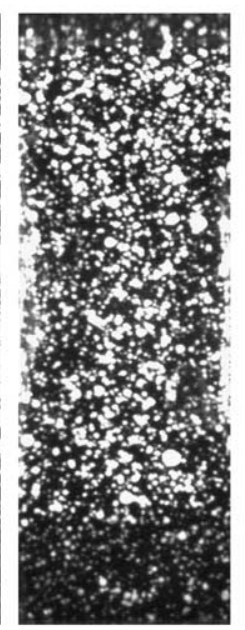

A2

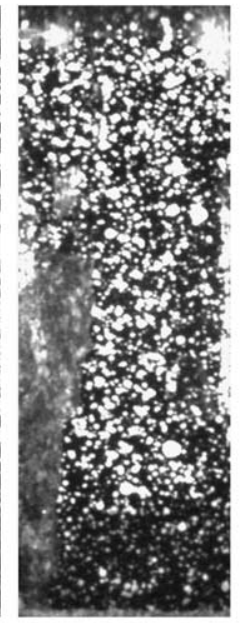

A6

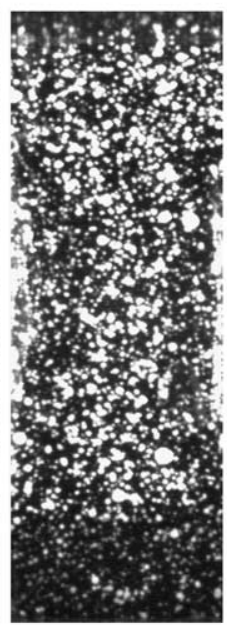

A3

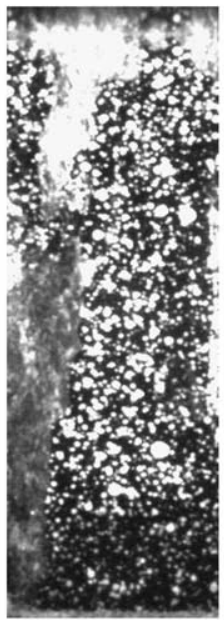

A7

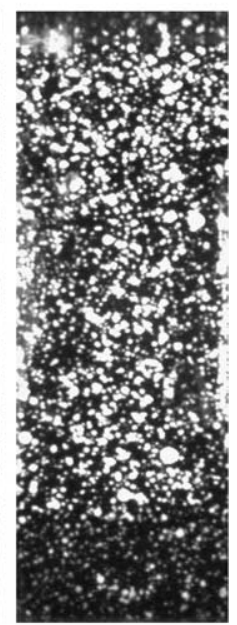

A4

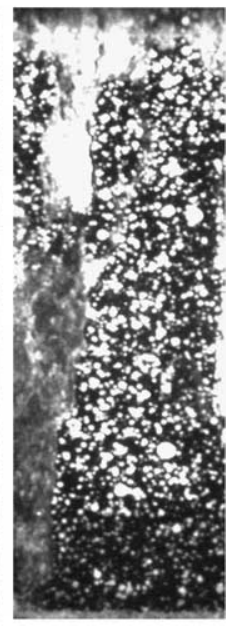

A8

Fig. 4. Eight evolution patterns of surface speckle image. Axis 2 indicates the loading direction and axis 1 the direction vertical to loading.

\subsection{Synchronization of multi-scale observations}

Furthermore, the main boards of the computers, which control the AE system and image capture system respectively, are installed with A/D cards. The key point in synchronization is that the load signal recorded by the MTS810 testing machine is transferred to the A/D cards in the image capture and acoustic emission systems respectively (Fig. 2). In this way, the load-time signal is recorded by the three experimental systems respectively (see Fig. 7(a)). Obviously, the three loadtime curves should collapse into one curve after sliding them along time axis (Fig. 7(b)). Correspondingly, after sliding the surface image series and acoustic emission series along the time axis with the same value as the load-time curves, the synchronization of the responses obtained by the three experimental systems is realized. Therefore, the responses of the sample at different scales, 

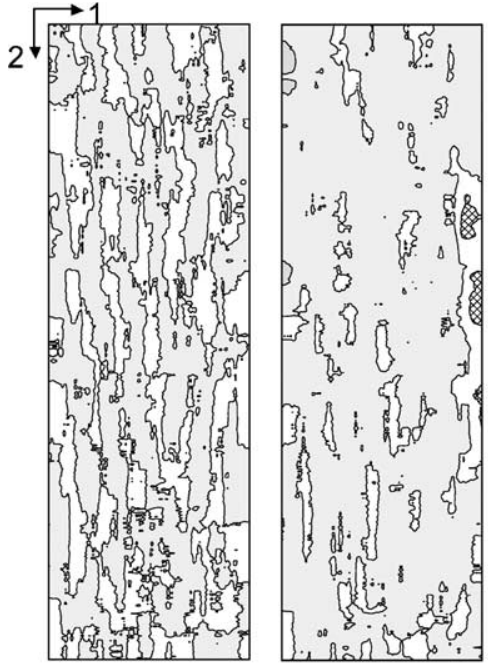

A2

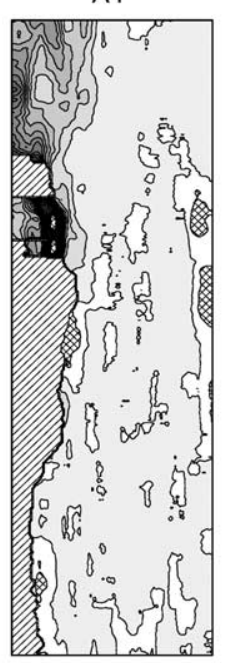

A5

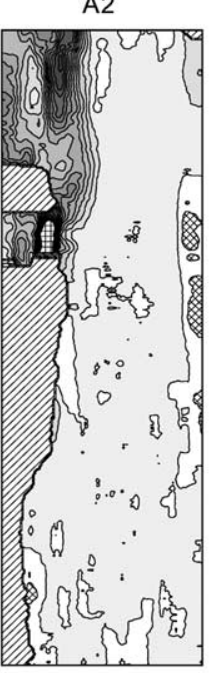

A6

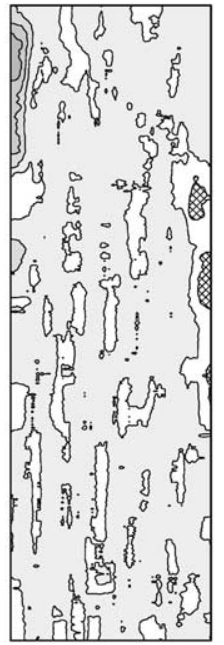

A3

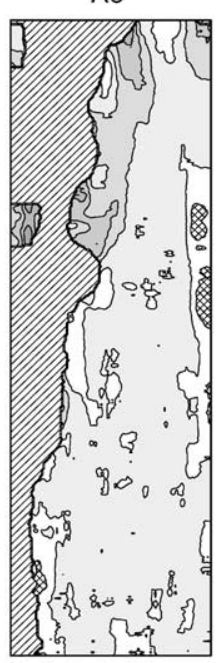

A7

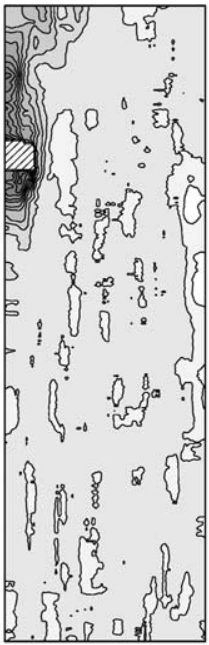

A4

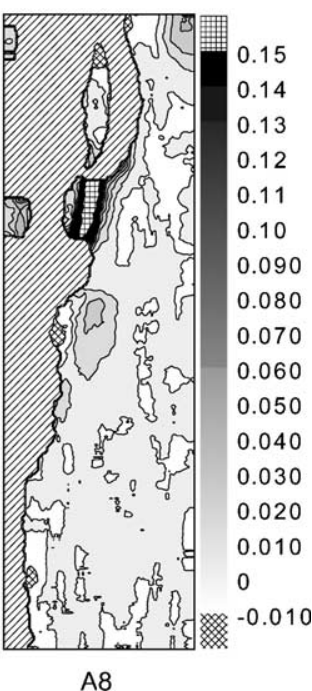

Fig. 5. Contour maps of surface strain field $\varepsilon_{11}^{*}$ relative to the initial image pattern, corresponding to the speckle patterns marked by the same letters in Fig. 4. WI indicates that the area where DSCM is not effective since the deformation is too big or the surface speckles fall off.

such as macroscopic nominal stress-strain curve, surface strain series at locally macroscopic scale, and acoustic emission series owing to microcracks at mesoscopic scale, can be analyzed synchronously.

\subsection{Speed-shift image capture}

However, in the present experiment, a loading process will last longer than $10 \mathrm{~min}$. If the image is captured with the maximum image capture speed of the CCD, 25 images per second, there will be more than 15,000 images. This will be huge workload for storage and data processing. If we use a unique low image capture speed, some useful details might not be obtained, especially near rupture, since the deformation develops very fast at this stage. Thus, this becomes a critical problem in the synchronous experiment. After considering the deformation to be stable in the early loading 

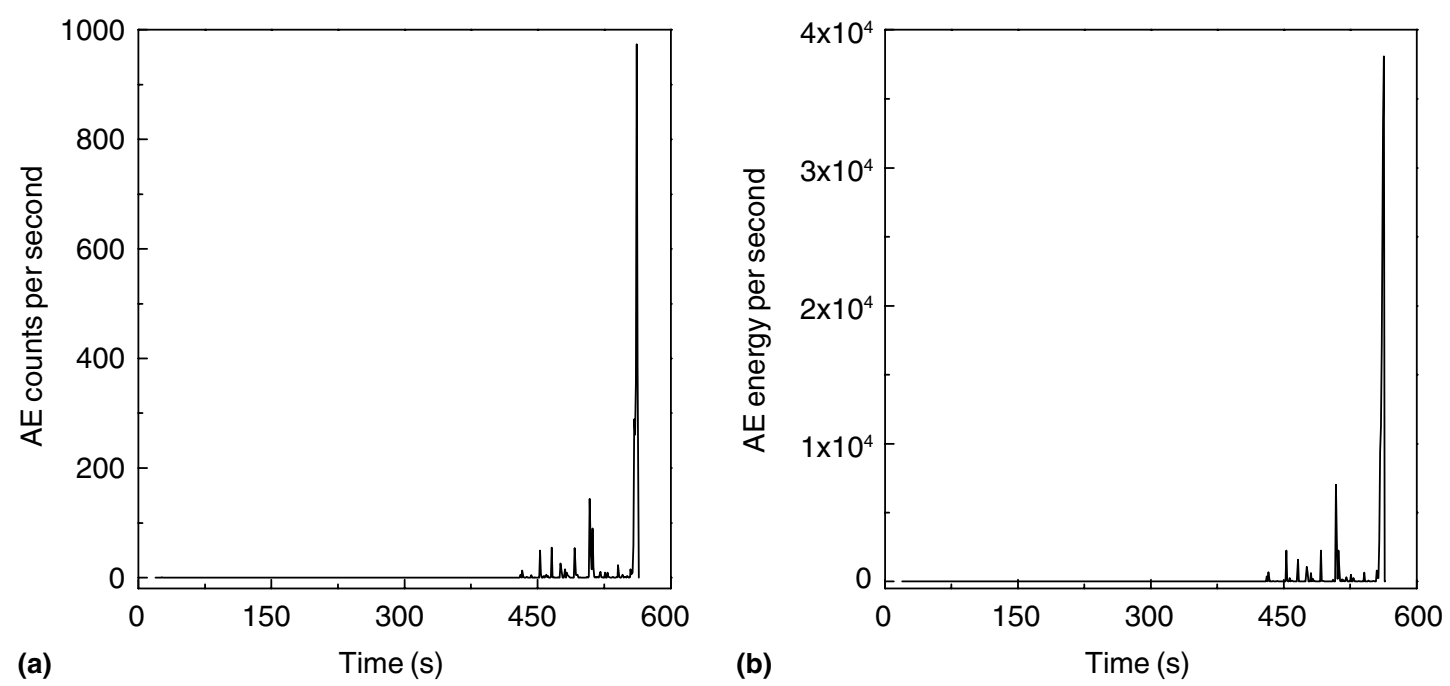

Fig. 6. (a) AE counts series. (b) AE energy series.
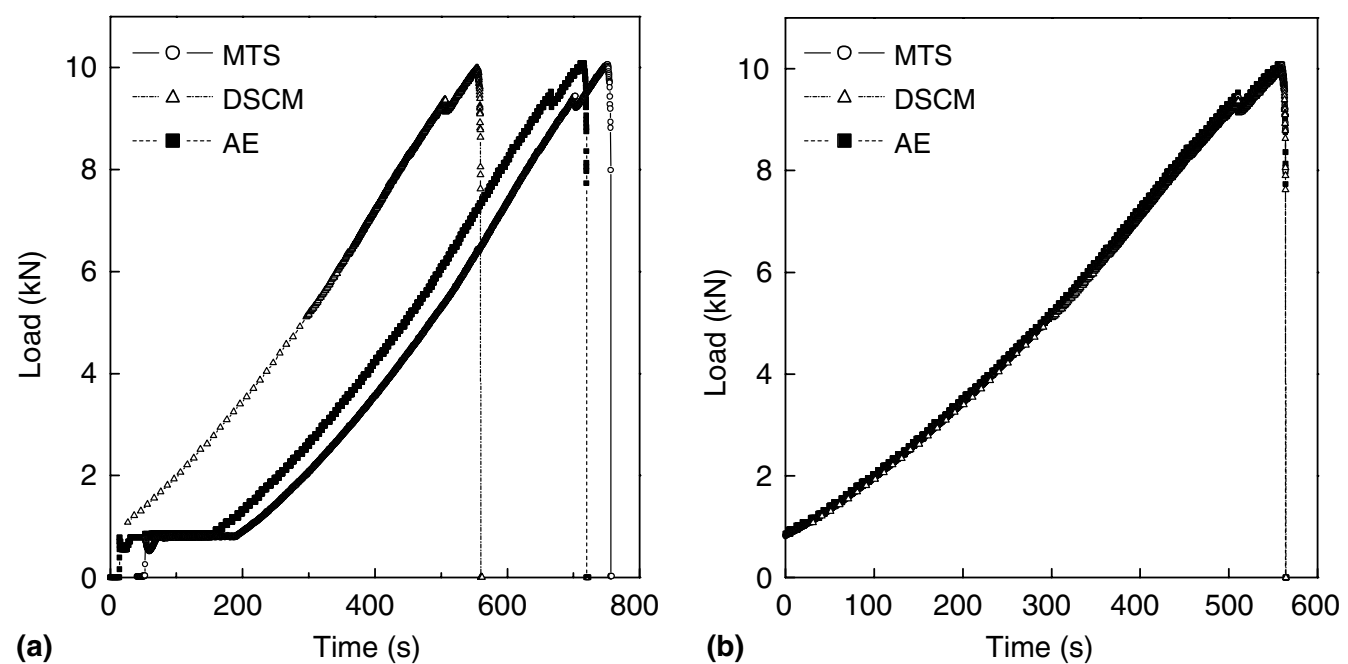

Fig. 7. Curves of load versus time captured by MTS810 ( $\bigcirc$ and corresponding line), DSCM ( $\triangle$ and corresponding line), and AE ( $\square$ and corresponding line) system respectively. (a) Before synchronous processing. (b) After synchronous processing.

stage while change much faster at later stage, we adopt a speed-shift image capture method controlled by the external load.

Based on some preliminary experiments, we adopt the image capture speed as follows: the speckle images are captured with time interval of $10 \mathrm{~s}$ when load is less than $5 \mathrm{kN}, 1 \mathrm{~s}$ when load is between $5 \mathrm{kN}$ and $6 \mathrm{kN}, 500 \mathrm{~ms}$ between $6 \mathrm{kN}$ and
$6.5 \mathrm{kN}$, and $80 \mathrm{~ms}$ when load is greater than $6.5 \mathrm{kN}$ (Fig. 8). The comparison of the capture speeds of the designed (dashed line) and the actually realized (solid line) indicates that the speed-shift image capture method can be satisfactorily realized. The control of image capture speed ensures that the system can capture enough useful images with the current image capturing equipment. 


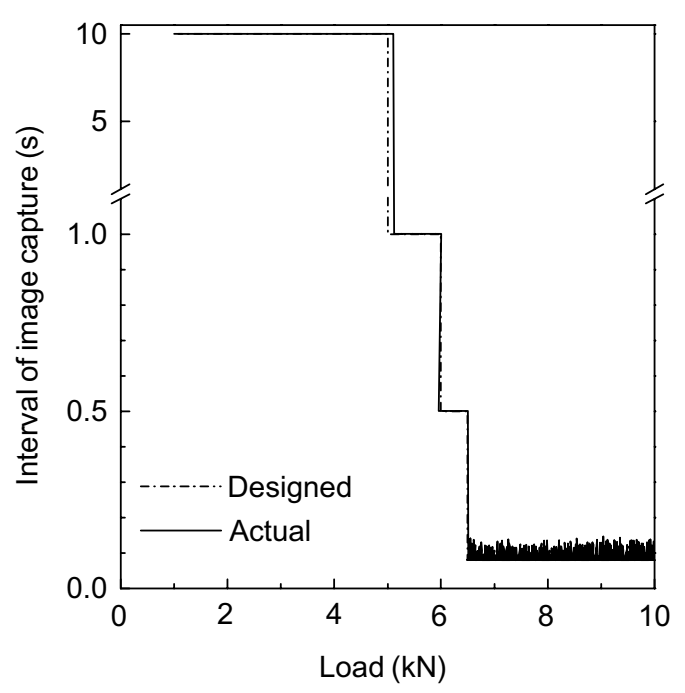

Fig. 8. Image capture interval versus load on rock sample, actual captured curve by the system (solid line) and designed curve (dashed line).

\section{Experimental results}

\subsection{Synchronous observations of the damage and rupture}

Based on the synchronization of the three experimental systems, we can synchronously draw various responses at different scales with the same variable-nominal strain $\varepsilon^{*}$, such as nominal stress $\sigma^{*}$ (Fig. 9, solid line), global mean damage fraction $D$ (Fig. 9, $\mathbf{\square}$ and corresponding line, where points A1-A8 (|) represent the eight evolution patterns shown in Figs. 4 and 5), and cumulative AE energy $\Theta^{*}$ (Fig. 9, and $\square$ corresponding line). In data processing, $D$ is calculated from the experimental nominal stress $\sigma^{*}$ and strain $\varepsilon^{*}$ curve [10], and $\Theta^{*}$ is obtained from the AE energy series.

It can be seen that the damage-failure process of the gabbro sample under uniaxial compression has three phases:

(1) The first phase (from A1 to A2 in Fig. 9): At the initial loading stage (much before A2), there is no AE signal since the events of mesoscopic damage are too small to be detected by AE sensors. The surface strain field is nearly homogeneous (A1 in Fig. 5) and the

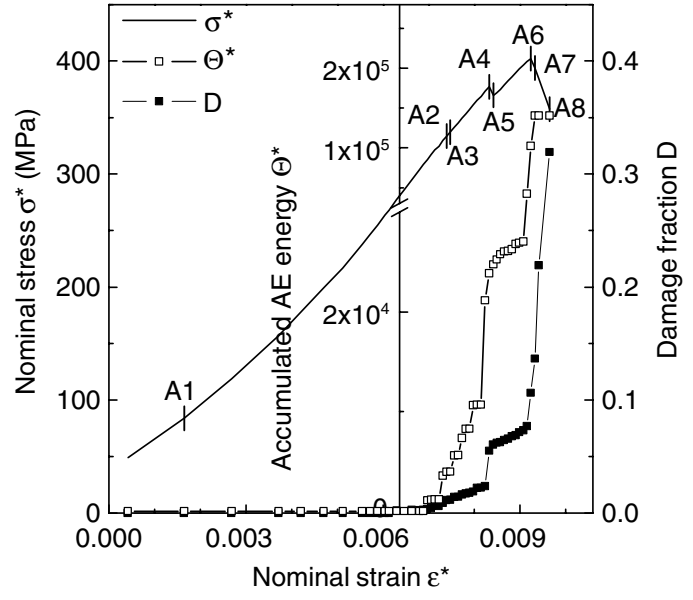

Fig. 9. Curves of nominal strain $\varepsilon^{*}$ versus nominal stress $\sigma^{*}$ (solid line), mean damage fraction $D$ ( $\boldsymbol{\square}$ and corresponding line), points $\mathrm{A} 1-\mathrm{A} 8(\mid)$ represent the eight evolution patterns indicate in Figs. 4 and 5, and cumulative AE energy $\Theta^{*}$ ( $\square$ and corresponding line).

mean damage fraction is nearly zero. When approaching to A2, AE signals appear. This indicates that micro-damage has become stronger and can be detected by the AE system. Meanwhile, surface strain field is still approximately homogeneous (A2 in Fig. 5), only more tensile strain domain than A1 owing to transverse effect. Also, the mean damage fraction remains a low level.

Obviously, the nucleation, growth and minor coalescence of micro-damage happen here and there, but the rock sample remains globally stable in this phase.

(2) The second phase (from A2 to near A8 in Fig. 9): Beyond the first phase, $\Theta^{*}$ and $D$ increase faster, see A3 in Fig. 5. Correspondingly, a high-strain-gradient domain, which can be called strain localization, appears at the left-up corner of the surface strain field.

Afterwards, as load increases, there is a small drop in the nominal stress-strain curve (from A4 to A5 in Fig. 5), which is called small catastrophe macroscopically. Clearly, it can be seen that there is a jump in $\Theta^{*}$ and $D$, and the failure domain in the surface strain field extends to larger length scale. That is to say, the evolution of micro-damage causes 
obvious response on mesoscopic scale (AE energy release), locally macroscopic scale (strain localization and local failure), and macroscopic scale (mean damage fraction).

After the small catastrophe, it can be seen that the rock sample comes to a relatively stable state since $\Theta^{*}$ and $D$ increase much slower, and the strain localization domain increases a little only.

However, prior to the maximum nominal stress point (A6 in Fig. 5), the rock sample comes to another unstable status corresponding to another sharp increasing in the responses at different scales.In this phase, strain localization and other intermediatescale events, such as small catastrophe, appear.

(3) The third phase (A8 in Fig. 9): Eventually, the sample displays catastrophic rupture on sample scale.

Moreover, the AE energy distribution at different phases is shown in Fig. 10. It is found that the width of the energy distribution becomes wider with increasing load. And there is more large energy AE appears in the later loading phase than in the initial loading phase. This indicates that many small cracks occur in the initial loading stage while more larger cracks in the eventual rupture.

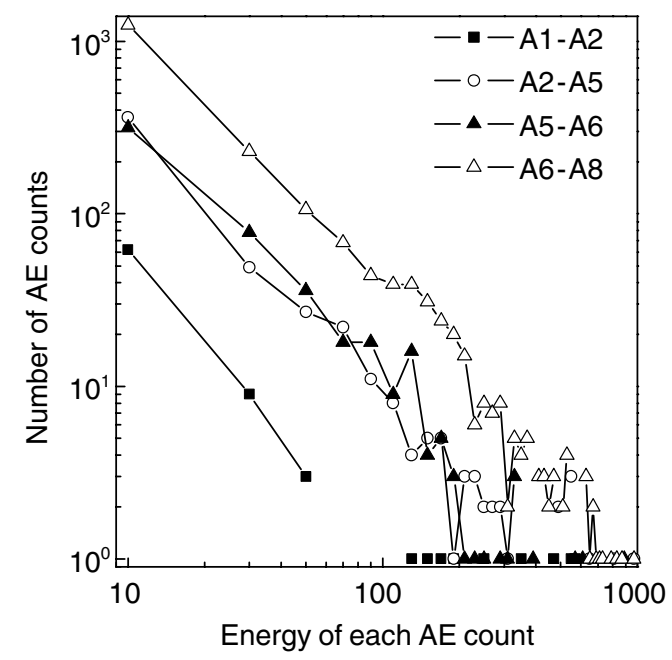

Fig. 10. Distribution of acoustic emission energy at different loading phase.

\subsection{Critical sensitivity on multiple scales}

More interestingly, the synchronous multi-scale observations of rock damage and rupture provide pronounced trans-scale effects of rock on failure. In order to combine the macroscopic external controlling variable, i.e. boundary displacement $U$, with the responses $R$ of the rock sample at different scales, the concept of sensitivity [11] is adopted. Here, we define sensitivity $S$ as

$S=\frac{\Delta}{\Delta U}\left(\frac{\Delta R}{\Delta U}\right)$,

critical sensitivity means that responses at different scales to the controlling variable, i.e. boundary displacement $U$, may increase significantly, i.e. $S \gg 1$, prior to catastrophe.

In this paper, we adopt the responses at three different scales, i.e. the mean damage fraction $D$ at macroscopic scale, the distance $\Delta H^{*}$ between successive patterns of surface strain at locally macroscopic scale and the cumulative AE energy $\Theta^{*}$ owing to micro-cracks at mesoscopic scale. In data processing, $\Delta H^{*}$ can be calculated from the surface strain patterns,

$\Delta H^{*}=\frac{1}{N_{\text {eff }}} \sum_{x=1}^{131} \sum_{y=1}^{401}\left|\Delta \varepsilon_{i j}^{*}(x, y)\right|, \quad i, j=1,2$,

where $\Delta \varepsilon_{i j}^{*}(x, y)$ is the increment of strain tensor $\varepsilon_{i j}^{*}(x, y)$ at point $(x, y), 131$ and 401 are the totals of calculating points in the strain field along axis 1 (loading direction) and axis 2 (vertical to loading) respectively, and $N_{\text {eff }}$ is the effective points, i.e. effective for DSCM calculation.

Now, we have had sensitivity $S$ at the three scales, $S_{D}$ from macroscopic damage, $S_{H^{*}}$ from locally macroscopic surface strain patterns and $S_{\Theta^{*}}$ from mesoscopic AE. Fig. 11 shows the sensitivities $S$ at the three scales for a sample. At the initial stage, $S_{\Theta^{*}}$ is equal to zero since the events of mesoscopic damage are too small to be detected by AE sensors, $S_{H^{*}}$ and $S_{D}$ are also equal to zero since the change of the deformation patterns is nearly zero and macroscopic damage can be neglected at the initial stage. In other words, at the initial stage, the responses of the system at all scales are not sensitive to the external controlling variable, i.e. the boundary displacement. As the boundary 

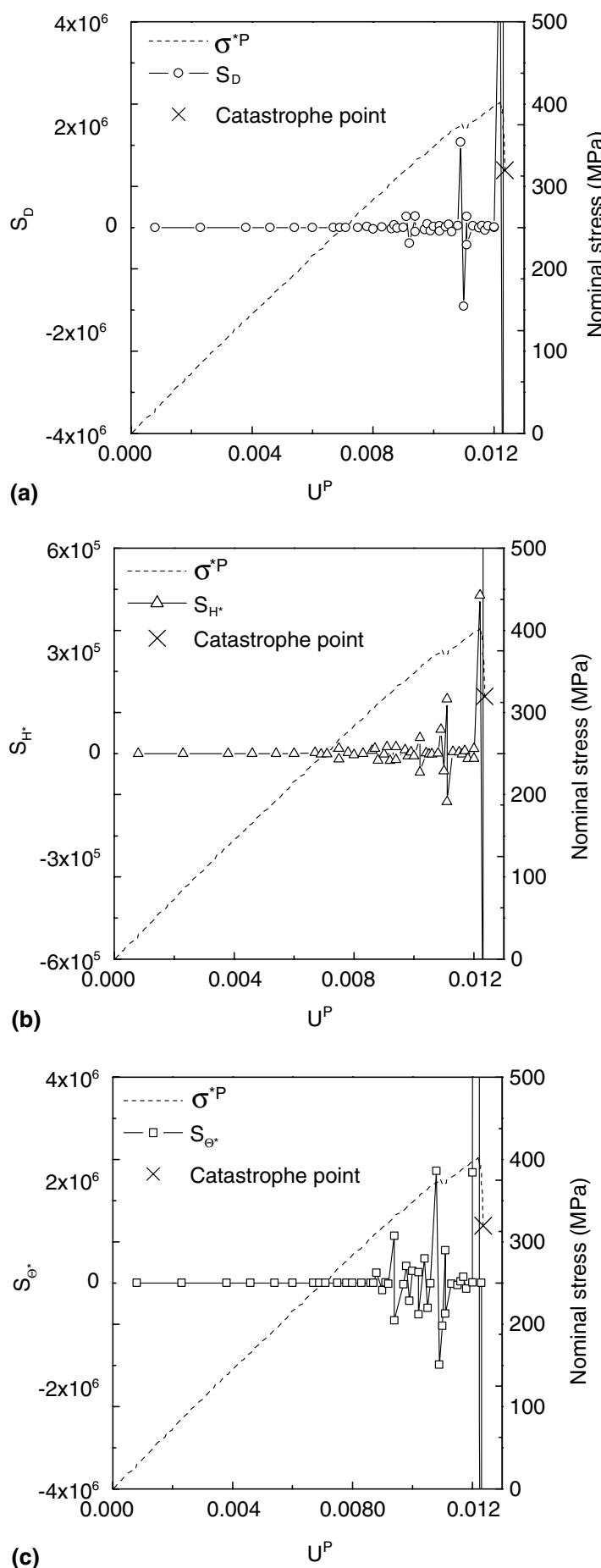

Fig. 11. (a) Sensitivity $S_{D}$ calculated from damage. (b) Sensitivity $S_{H^{*}}$ calculated from surface strain patterns. (c) Sensitivity $S_{\Theta^{*}}$ calculated from released elastic energy. displacement increases, the three sensitivities keep in low level. This means that the system is in a state with low sensitivity. However, the three sensitivities $S_{\Theta^{*}}, S_{H^{*}}$, and $S_{D}$, present oscillations one after another (Fig. 11) with increasing load and increases significantly near the catastrophe transition point. This implies that the system becomes highly sensitive prior to catastrophe point, from mesoscopic scale to macroscopic scale. This is what we called critical sensitivity on multiple scales. Noticeably, the critical sensitivities of AE energy, surface strain patterns and mean damage to the external boundary displacement demonstrate the linking of several scales: from mesoscopic events to macroscopic behavior. So, such sensitivities imply that a minor change on microscopic level can induce significant cascade effect, from mesoscopic to macroscopic scales as the system approaches the catastrophe.

\subsection{Trans-scale fluctuations}

Furthermore, trans-scale fluctuations of the strain pattern from $10^{-5}$ sample scale to larger scale were revealed by coarse-grained average on strain patterns. The approach is as follows: Firstly, set a window with size $N \times N$ (here, we adopt $N$ as an odd number) pixels around a point $(x, y)$ in the surface strain field, then take the mean value of the points in the window,

$$
\begin{gathered}
\overline{\varepsilon_{i j}^{*}}(x, y) \\
=\sqrt{\frac{1}{N^{2}} \sum_{m=1}^{N} \sum_{n=1}^{N} \varepsilon_{i j}^{*}\left(x-\frac{N+1}{2}+m, y-\frac{N+1}{2}+n\right)}, \\
i, j=1,2
\end{gathered}
$$

as the strain at the point $(x, y)$ on scale $N \times N$. Secondly, after sliding the window on the whole strain field, a strain field at spatial level of $N \times N$ is obtained. Finally, the standard deviation,

$$
\delta_{N}\left(\varepsilon_{i j}^{*}\right)=\sqrt{\frac{1}{N_{\text {eff }}^{\prime}} \sum_{x=1}^{132-N} \sum_{y=1}^{402-N}\left[\overline{\varepsilon_{i j}^{*}}(x, y)-\overline{\overline{\varepsilon_{i j}^{*}}}\right]^{2}}
$$$$
i, j=1,2,
$$

can be assumed as the fluctuation of the strain field at scale $N \times N$, where $\overline{\overline{\varepsilon_{i j}^{*}}}$ is the mean value of the 
whole strain field at level $N \times N$, and $N_{\text {eff }}^{\prime}$ is the corresponding effective points number in such a strain field.

In the present study, set the windows $1 \times 1$, $9 \times 9,31 \times 31$, and $101 \times 101$ pixels, which correspond to about $10^{-5}, 10^{-3}, 10^{-2}$, and $10^{-1}$ sample scale. The fluctuations of strain field $\varepsilon_{11}^{*}$ on the four scales versus nominal strain $\varepsilon^{*}$ are shown in Fig. 12. It is clear that during the whole loading process, the fluctuations decrease as the scale increase. At the initial stage (before A2), fluctuations at different scales keep in a low level, no more than 0.002 , and the corresponding strain field remains nearly homogeneous (A1 and A2 in Fig. 5). However, prior to the main rupture the fluctuations at all scales increase significantly, which signifies a very heterogeneous strain field (A3-A8 in Fig. 5). This is what we called trans-scale fluctuations [11]. Trans-scale fluctuations of strain field imply that the correlation length of strain increases progressively from small scale to large scale at the catastrophic rupture. Trans-scale fluctuations can be considered as a significant indication of the catastrophe transition and an immediate precursor

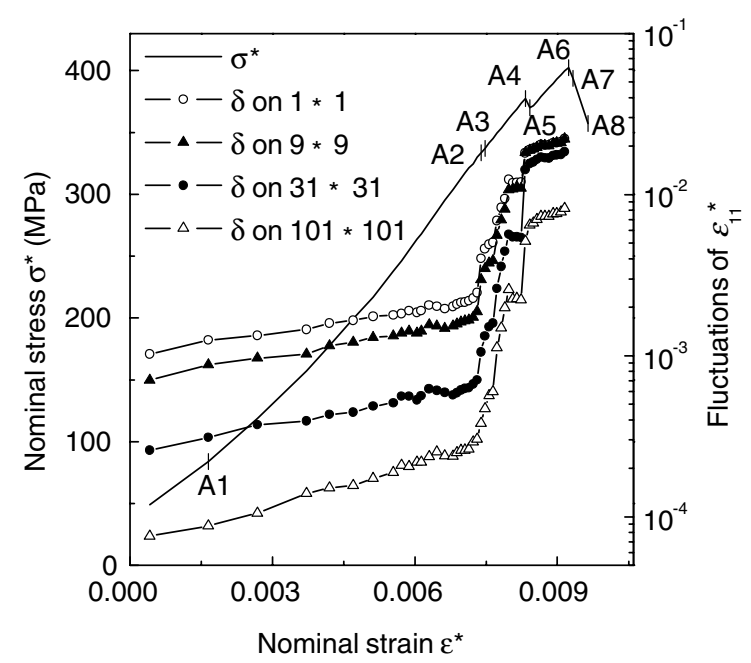

Fig. 12. Curves of nominal strain versus fluctuations of surface strain field $\delta_{N}\left(\varepsilon_{11}^{*}\right)$, coarse-grained on $1 \times 1$ ( $\bigcirc$ and corresponding line), $9 \times 9$ ( $\Delta$ and corresponding line), $31 \times 31$ ( corresponding line), and $101 \times 101(\triangle$ and corresponding line) pixels. Moreover, points A1-A8 (|) on the nominal stress-strain curve corresponding to the speckle patterns marked by the same letters in Fig. 4. to main rupture. This experimental result validates that trans-scale fluctuations might be a common precursor to catastrophe experimentally.

\section{Conclusions}

Multi-scale coupling plays an important role in damage and rupture of heterogeneous brittle media, such as rock. A synchronous multi-scale experimental system, consisting of MTS810 testing machine, DSCM, and AE technique, for rock damage and rupture is designed and realized. This provides an effective method for the study of such a complex problem.

The synchronous analysis of physical and mechanical responses of rock at different scales shows that there are significant correlation between damage evolution at small and large length scales, and the rupture at sample scale. Moreover, it is found that there are two common trends prior to catastrophe. One is critical sensitivity on multiple scales: the system becomes highly sensitive prior to catastrophe point, from mesoscopic scale to macroscopic scale. The other is trans-scale fluctuations: the correlation length of strain increases progressively from small scale to large scale at the catastrophic rupture. These experimental observations may provide some useful information for understanding the physics underlying catastrophic rupture in rock.

\section{Acknowledgements}

This work is supported by the National Natural Science Foundation of China (Grant No. 10172084, 10232050, 10372012 and 10472118), the Major State Research Project "Nonlinear Science" G200007735. The authors would like to thank Haisheng Yang and Chengyu Gu for helpful works in the experiments.

\section{References}

[1] D.R. Curran, L. Seaman, D.A. Shockey, Dynamic failure of solids, Phys. Rep. 147 (1997) 253-388. 
[2] Z.P. Bazant, E.P. Chen, Scaling of structural failure, Appl. Mech. Rev. 50 (1997) 593-627.

[3] Y.L. Bai, C.S. Lu, F.J. Ke, M.F. Xia, Evolution induced catastrophe, Phys. Lett. A 185 (1994) 196-200.

[4] M.F. Xia, F.J. Ke, Y.J. Wei, J. Bai, Y.L. Bai, Evolution induced catastrophe in a nonlinear dynamical model of material failure, Nonlinear Dyn. 22 (2000) 205224.

[5] F. Rong, H.Y. Wang, M.F. Xia, F.J. Ke, Y.L. Bai, Damage coalescence induced catastrophic rupture in heterogeneous brittle media, Pure Appl. Geophys., submitted for publication.

[6] S.P. Ma, X.H. Xu, Y.H. Zhao, The geo-DSCM system and its application to the deformation measurement of rock materials, Int. J. Rock Mech. Mining Sci. 41 (2004) 411412 .
[7] W.H. Peter, W.F. Randson, Digital imaging techniques in experimental stress analysis, Opt. Eng. 21 (1981) 427-431.

[8] D.A. Lockner, J.D. Byerlee, V. Kuksenko, A. Ponomarev, A. Sidorin, Quasi-static fault growth and shear fracture energy in granite, Nature 350 (1991) 39-42.

[9] X.L. Lei, K. Kusunose, M.V.M.S. Rao, O. Nishizawa, T. Satoh, Quasi-static fault growth and cracking in homogeneous brittle rock under triaxial compression using acoustic emission monitoring, J. Geophys. Res. 105 (2000) 6127-6139.

[10] X.H. Xu, M.F. Xia, F.J. Ke, Y.L. Bai, Critical sensitivity in rock experiments, Pure Appl. Geophys., accepted for publication.

[11] M.F. Xia, Y.J. Wei, F.J. Ke, Y.L. Bai, Critical sensitivity and trans-scale fluctuations in catastrophe rupture, Pure Appl. Geophys. 159 (2002) 2491-2509. 